\title{
Application of neural network for real-time measurement of electrical resistivity in cold crucible
}

\author{
Pavel Votava, Igor Poznyak *
}

\begin{abstract}
The article describes use of an Induction furnace with cold crucible as a tool for real-time measurement of a melted material electrical resistivity. The measurement is based on an inverse problem solution of a $2 \mathrm{D}$ mathematical model, possibly implementable in a microcontroller or a FPGA in a form of a neural network. The 2D mathematical model results has been provided as a training set for the neural network. At the end, the implementation results are discussed together with uncertainty of measurement, which is done by the neural network implementation itself.
\end{abstract}

Keywords: induction melting, melted material resistivity measurement, neural network

\section{Introduction}

An induction furnace with cold crucible is a complete device for melting any electrically conductive material. The principle of its operation is based on a direct application of the Lenz law and the Joule effect, whereby induced eddy currents heat the material placed in alternating magnetic field. These technologies are used for high-temperature liquid-phase synthesis of oxides, ceramics and glasses with temperatures ranging up to $3000{ }^{\circ} \mathrm{C}$, [1]. As has been theoretically and experimentally verified [2], the same installations can be used for contactless measurement of electrical resistivity of the melted material.

However, these measurements are based on inverse problem solution of electromagnetic mathematical models, whose calculation takes usually several minutes even on powerful workstations, depending on the model and its required accuracy. Therefore, the resistivity assessments are performed after completion of the melting processes. The issue occurs when the measurement system is supposed to have a real-time response, because several different setups of mathematical model needs to be calculated regarding the inverse problem solution. In addition to that, at least two approaches should be implemented to be able to provide the solution in a full measuring scale [3].

The implementation of a neural network capable to provide the inverse problem solution, based on a specified training set calculated by electromagnetic mathematical model, presents a possibility of getting the online results in the real time during the experiment. The optimally trained and set-up neural network can successfully calculate the results for a wide range of a different geometric and electrical parameters of the induction furnace, in a very short time and can be easily implemented in a microcontroller- or FPGA- based measurement system.

\section{Theoretical background}

The measurement of molten material electrical resistivity is based on comparing the experimentally measured values of induction system (IS) parameters with calculated values of the chosen mathematical model (MM). Figure 1 shows this situation for power balance curve approach, based on measurement of the total power of the IS and a recalculation of its power distribution from MM as a function of melted material electrical resistivity. The measured value can be determined by multiplication of absolute values of inductor voltage, current and power factor, as figure 1 shows. The MM solution is obtained as a sum of power losses in the cold crucible walls, its bottom and collar, the molten material and the inductor, respectively.

Every power balance curve mentioned in this article is shown as a function of parameter $\mathrm{m} 3$, which is defined as the ratio of melted material radius to its penetration depth, as the equation 1 shows. The multiplication factor of 2 has been used in the formula, as the final shape of balance curves is smoother, which is significantly better for neural network training. The parameter is used mainly because of its better balance curve distribution in comparison with resistivity itself. The electrical resistivity ? increases monotonically in a range of $j 0.0002 ; 1.5$ i $\Omega \mathrm{cm}$, which is expected fit for investigated oxides. This monotonic increase is a main principle of resistivity measurement and it is assumed in every following chart representation. As shown in figure 1, the total power distribution is calculated by the MM for every single geometrical and electrical setup for whole mentioned resistivity range and its final value is obtained by inverse problem solution based on power measurement

$$
\left.m_{3}\right|_{\delta_{\text {melt }}}=\sqrt{2\left(r_{\text {melt }} / \delta_{\text {melt }}\right)}
$$

* Department of Electric power engineering and Ecology, University of West Bohemia, Pilsen, Czech Republic, flow85@kee.zcu.cz, poznyak@fst.zcu.cz 


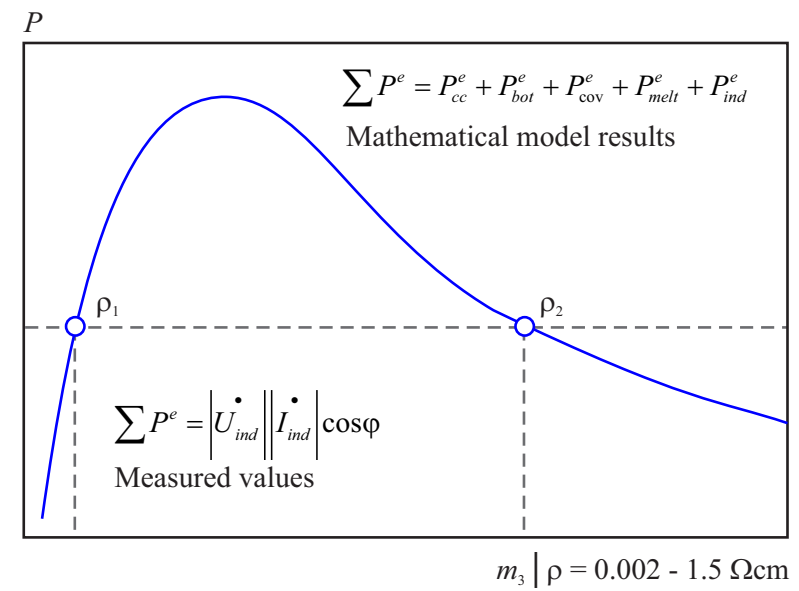

Fig. 1. Power balance curve as a function of resistivity parameter $m_{3},[3]$

Figure 1 also shows the two possible solutions, whereby only one of them is real. Therefore, an additional condition has to be applied [4]. In addition, a second approach of measurement should be used in parallel to the first one, because an uncertainty of measurement is functionally dependent on the derivative of the balance curve, as shown in Figure 2. Power balance approach has no solution in the area of zero derivative and its uncertainty is rising to maximum in the surrounding. Therefore an approach based on the impedance of the induction system has been established.

Figure 2 shows normalized curves of power balance approach, impedance approach and its uncertainty with the red marked area showing the highest accuracy region. From the picture it is clear that both approaches have to be used to obtain the most accurate solution in the full range, together with appropriate number of MM solutions for the required accuracy.

Obtaining the smooth balance curves from the MM is a time-consuming process, which could be only barely implemented in the real time system. The System requirements are strongly dependent on MM parameters, layout of the IS, required accuracy and time response. In our case a 2D MM has been chosen that can be described by (2) to (4) while taking into account a quasi-stationary field, where $\gamma$ is an inverse value of electrical resistivity, which is obtained as the inverse problem solution from its progression range [4]. A single-valued electrical resistivity has been taken into account, due to inverse problem solution, in spite of temperature variations in the melt. This can cause significantly higher error in calculation, especially for higher values of parameter $m_{3}$

$$
\begin{gathered}
\frac{1}{\mu_{0}} \Delta \dot{A}-j \omega \gamma \dot{A}=\dot{J}_{\Omega 1}+\dot{J}_{\Omega 34}, \\
\dot{J}_{\Omega 34}=-j \omega \gamma \dot{A}, \\
\operatorname{div}\left(\dot{J}_{\Omega 34}\right)=0 .
\end{gathered}
$$

Figure 3 represents a draft of the 2D MM, which assumes an axially-symmetric geometry, where the cold crucible walls, the inductor and the collar widths have been

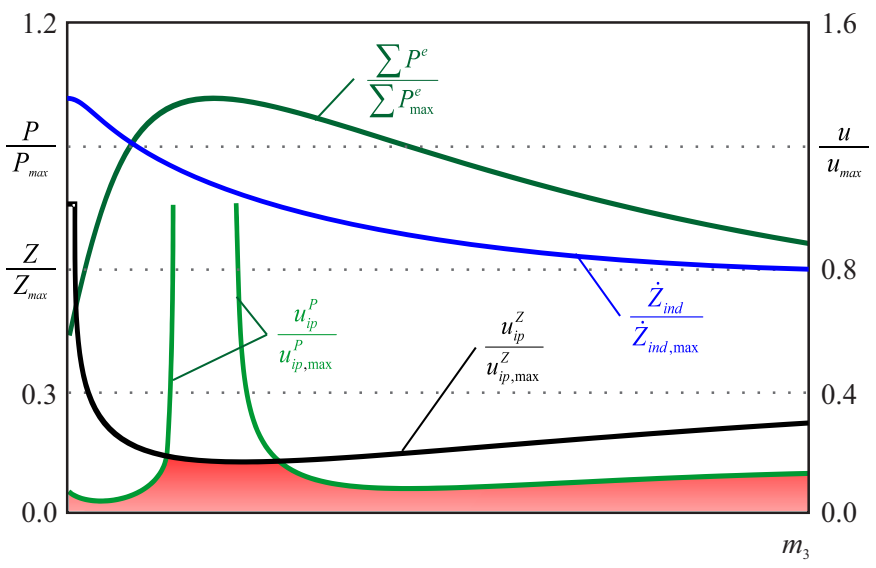

Fig. 2. Normalized power balance and impedance curves as a function of resistivity and corresponding uncertainty [4]

reduced to penetration depth width, because of simplification and computational time reduction. Detailed information about MM can be found in [2] and [5].

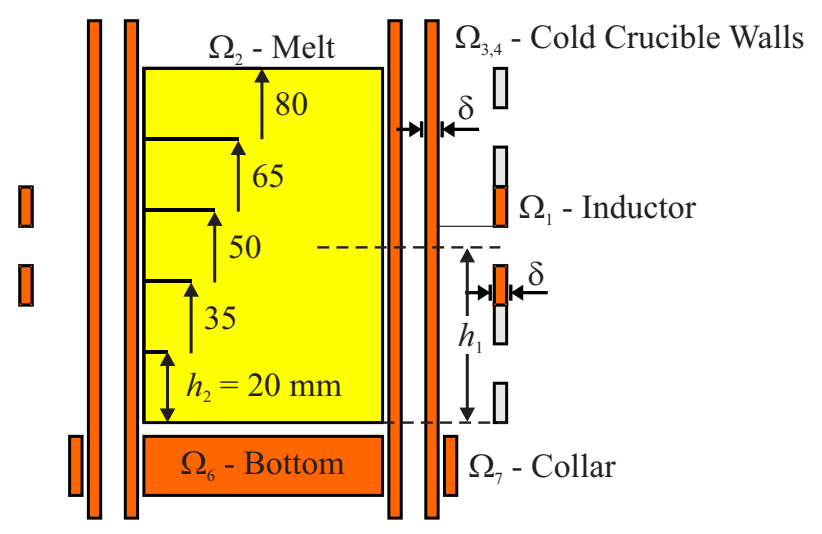

Fig. 3. Draft of the $2 \mathrm{D}$ mathematical model

Table 1. MM investigation setup

\begin{tabular}{lcc}
\hline$h_{2}(\mathrm{~mm})$ & $h_{1}(\mathrm{~mm})$ & $f(\mathrm{MHz})$ \\
\hline 20 & $-10.0-$ & 0.500 \\
35 & $-17.5-$ & 1.000 \\
50 & $15.0,25.0,35.0$ & 2.000 \\
65 & $15.0,32.5,50.0$ & 3.858 \\
80 & $15.0,40.0,65.0$ & - \\
\hline
\end{tabular}

\section{Mathematical model characterization}

For investigating of the IS behavior several solutions of MM have been calculated, based on different geometrical arrangement and electrical parameters. The IS layouts are indicated in Fig. 3, where variable parameters are the position of the inductor $h_{1}$ relatively to the cold crucible bottom and the melted material height $h_{2}$. Complete setup is shown in Tab. 1, together with frequency step 


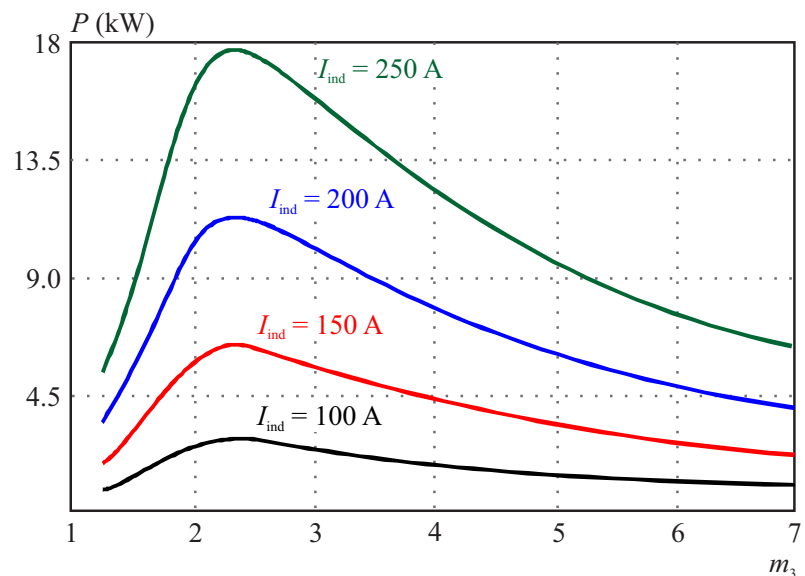

Fig. 4. Power balance curves as a function of resistivity parameter $\mathrm{m} 3$, for different values of $I_{\text {ind }}$

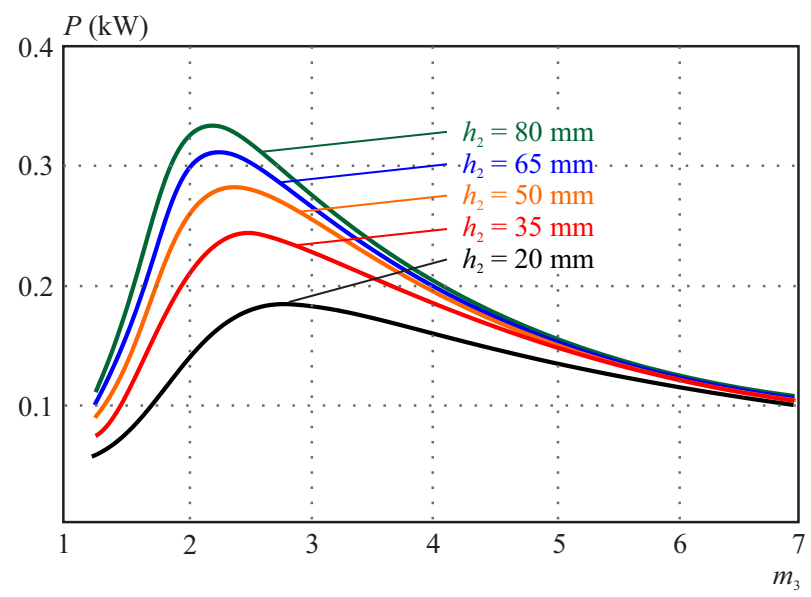

Fig. 6. Power balance curves as a function of resistivity ratio $m_{3}$, for different values of $h_{2}$

$f$ used for every geometrical combination. The range of frequencies has been selected based on the availability of generators used for melting in the Research Centre.

In total, 11 different geometrical arrangements at 4 chosen frequencies have been solved. For every setup, 22 values of parameter $m_{3}$, and thus of electrical resistivity, have been calculated. The explored range of the $m_{3}$, parameter is from 0.4 to 7 with 0.3 step. It means 968 MM solutions that have been furthermore interpolated to obtain a final set of 215006 results.

The only electrical variable, from a neural network input parameters point of view, in the MM is the frequency, because the power distribution ratio in the IS parts is not affected by changing the other parameters such as current and voltage of the inductor. The electrical resistivity of melted material had been changed in mentioned range of MM investigation as well, however it doesn't represent an input variable, but the output solution. This statement has been verified by graphically in Fig. 4 and Fig. 5, where the impedance of the IS shown to be independent on $I_{\text {ind }}$ and the total power $P$ can be recalculated for any current

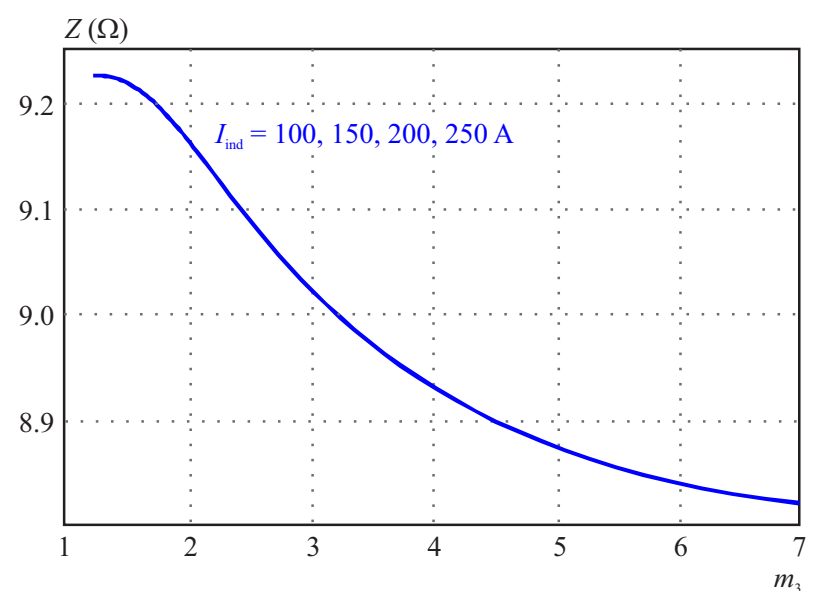

Fig. 5. Impedance curve as a function of resistivity ratio $\mathrm{m} 3$, for different values of $I_{\text {ind }}$

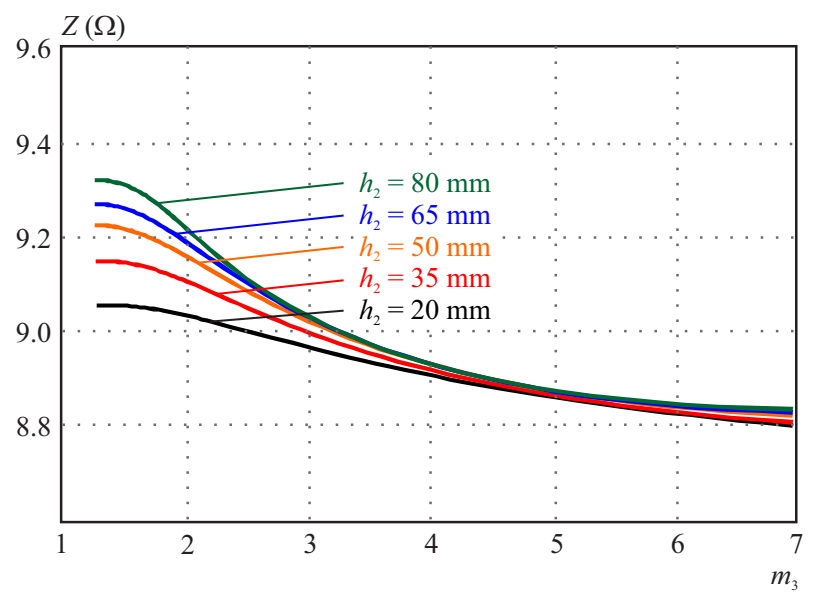

Fig. 7. Impedance balance curves as a function of resistivity ratio $m_{3}$, for different values of $h_{2}$

according to (5), of course only for the specific layout

$$
P_{2}=\frac{P_{1} I_{i n d, 1}^{2}}{I_{i n d, 2}^{2}}
$$

In addition, (5) allows us to normalize every setup and power balance curve to $I_{\text {ind }}=1 \mathrm{~A}$, which is more than convenient for implementation to any measurement system, because of its variable reduction. By this recalculation, not only the power distribution is normalized to the suitable range, but also the inductor current and voltage can be omitted from further calculations, because current becomes a constant and voltage is numerically equal to impedance.

Figures 6 and 7 show results of MM for variable heights of the melt with inductor in the middle position to it, for both power and impedance balance curves, respectively. Frequency is fixed at $3.8 \mathrm{MHz}$ and electrical resistivity parameter $m_{3}$ varies in the presented range. Very similar, but not negligibly different, balance curves can be obtained with fixed height of the melt and variable position of the inductor. Also, a frequency variation would show very similar results for a fixed layout of the IS. 


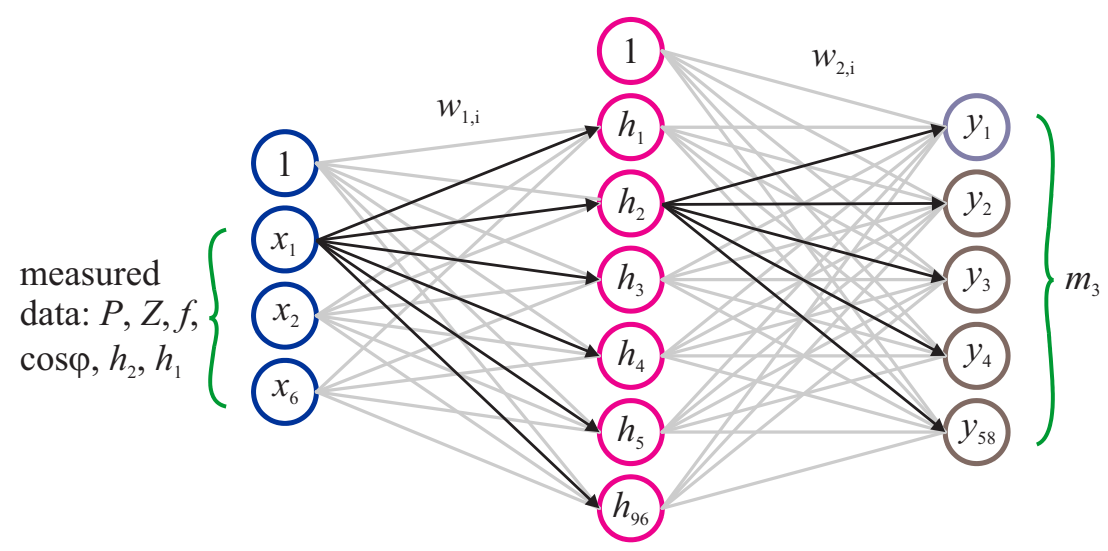

Fig. 8. Neural network arrangement

From the above described investigation of MM results, smooth output functions called balance curves have been found. They are dependent on geometrical and electrical parameters as height of the melt $h_{2}$, position of the inductor $h_{1}$, the total power $P$, IS impedance $Z$ and power factor $\cos \varphi$. Based on this, we can proceed to a neural network implementation, which is especially suitable in the case of smooth functions.

\section{Neural network implementation}

For final implementation, a 3-layer neural network $(\mathrm{NN})$ has been chosen, as shown in the figure 8 . The input layer contains measured parameters of the IS and a bias function, which is equal to 1 [6]. The hidden layer has 84 active neurons plus one bias neuron as well. Whereas there is only one output variable, parameter $m_{3}$, the output layer contains 58 neurons, because of its quantization. In principle, it means that 22 calculated values by the MM have been polynomially interpolated and requantized to 58 values. This procedure significantly saves computational time, yet maintains required accuracy.

Each neuron of the output layer represents a static value of the parameter $m_{3}$. According to it, a training set has been created, where each feature vector contains the input parameters and its matching output vector which contain only one activated output neuron at time. In this way, 2552 solutions based only on the MM results have been obtained, however this data have been furthermore interpolated in frequency with $100 \mathrm{kHz}$ step, to obtain more complex training set of 22330 feature vectors.

In this step, we have proceeded to Error-back-propagation learning algorithm [6], which is a common method of the NN training in combination with gradient descent optimization. Supervised learning is the process of setting weights $w$, shown in Fig. 8, with the objective of minimizing an output error represented by a cost function. The cost function $J(w)$ is defined by $(6)$, where $m$ is the feature vector quantity, $K$ is the number of input layer neurons, $S_{l}$ is the number of neurons in layer $l$

$$
\begin{aligned}
J(w)= & -\frac{1}{m}\left[\sum_{i=1}^{m} \sum_{k=1}^{K} y_{k}^{i} \log \left(h_{w}\left(x^{i}\right)\right)_{k}+\left(1-y_{k}^{i}\right) \times\right. \\
& \left.\times \log \left(1-\left(h_{w}\left(x^{i}\right)\right)_{k}\right)\right]+\frac{\lambda}{2 m} \sum_{l=1}^{l-1} \sum_{i=1}^{S l}\left(w_{i}^{l}\right)^{2} \\
& h_{w}(x) \in R^{K}
\end{aligned}
$$

The algorithm repeats the three-step cycle for every element of the training set: forward propagation, back propagation and weights update. Presented input vector is propagated forward through the NN, layer by layer, until it reaches the output layer. The actual output $y$ of the network is then compared to the desired output $c$, using the cost function, and an error value for each output neuron is calculated

$$
\begin{gathered}
\delta_{i}=f^{\prime}\left(y_{i}\right)\left(c_{i}-y_{i}\right), \\
\delta_{i}=f^{\prime}\left(h_{i}\right) \sum_{k=1}^{K} \delta_{k} w_{k, i} .
\end{gathered}
$$

The error values are then propagated backwards, until each neuron in each layer has an associated error value which roughly represents its contribution to the original output. Error signals for neurons in hidden layer are calculated by (8). The derivation of the activation function, in our case a binary sigmoid, is possible to obtain easily by

$$
f^{\prime}\left(y_{i}\right)=y_{i}\left(1-y_{i}\right) \text {. }
$$

The weight update, according to equation (10), is obtained as a sum of the actual weight value, plus multiplication of error signal with its input and a momentum, which can significantly speed up the learning process and is calculated as a multiplication of previous change of weight with constant $\alpha$. The coefficient $\rho$ is a step size, which also affects the speed of learning and is useful in the cost function global minimum searching, [6]

$$
w_{i}=w_{i}+\rho \delta_{i} x_{i}+\alpha \Delta w_{i}
$$




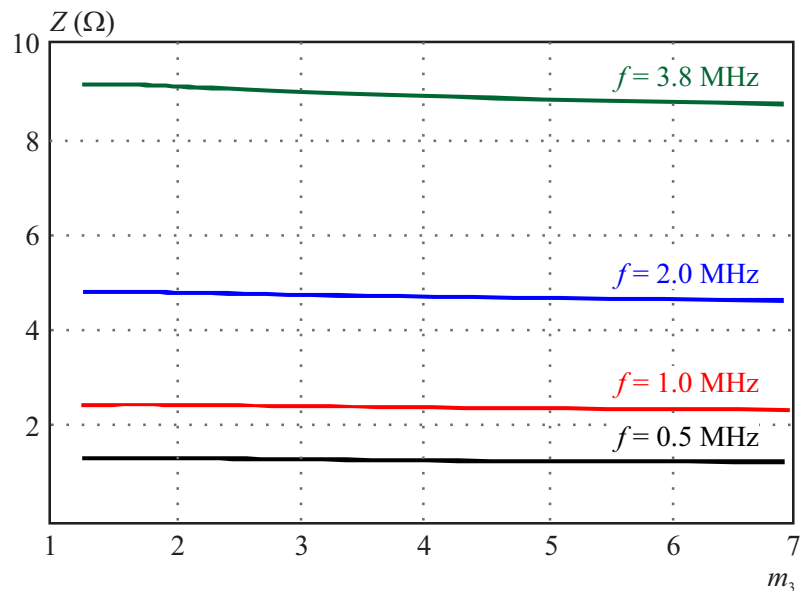

Fig. 9. Impedance as a function of resistivity ratio $m_{3}$, before the frequency normalization

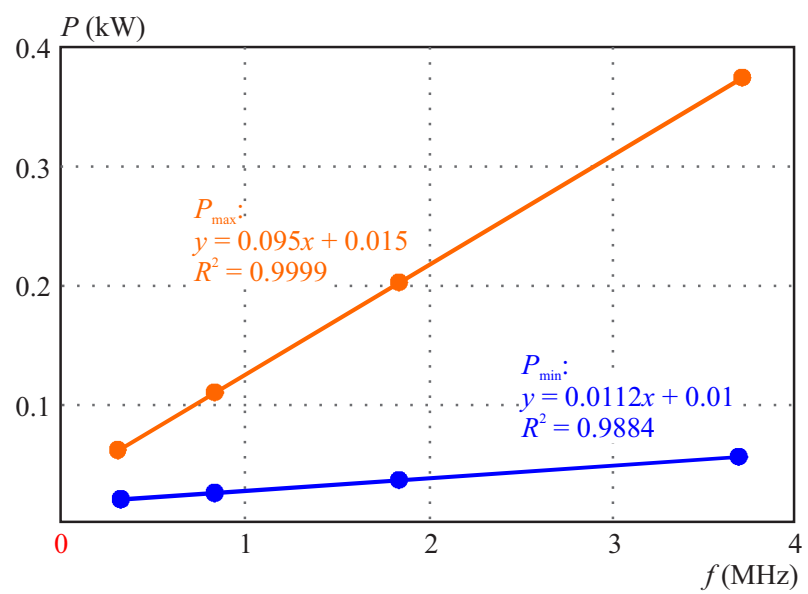

Fig. 11. Power-frequency data normalization

The importance of this process is that the neurons in the hidden layer organize themselves in a way that the different neurons learn to recognize different characteristics of the total input space. After training, when a new input pattern which has not been a part of the training set is presented to the NN, neurons in the hidden layer will respond with an active output if the new input contains a pattern that resembles a feature that the individual neurons have learned during their training.

The last step before the training process is a data normalization, which is very important because the input parameters have a different scale and learning process would not work that well. The large scale data would be significantly stronger in pattern recognition process and that would negatively affect the NN function. A linear transformation function, described by the following equation, has been chosen for the normalization purpose

$$
x_{i}=0.8 \frac{x-\min \left(x_{m}\right)}{\max \left(x_{m}\right)-\min \left(x_{m}\right)}+0.1 .
$$

This function sets the input data $\mathrm{x}$ into an interval $<0.1-0.9>$, however some input values are further scaled based on the input frequency, according to the Tab. 2 showing the minimal and maximal values of input parameters for its normalization.

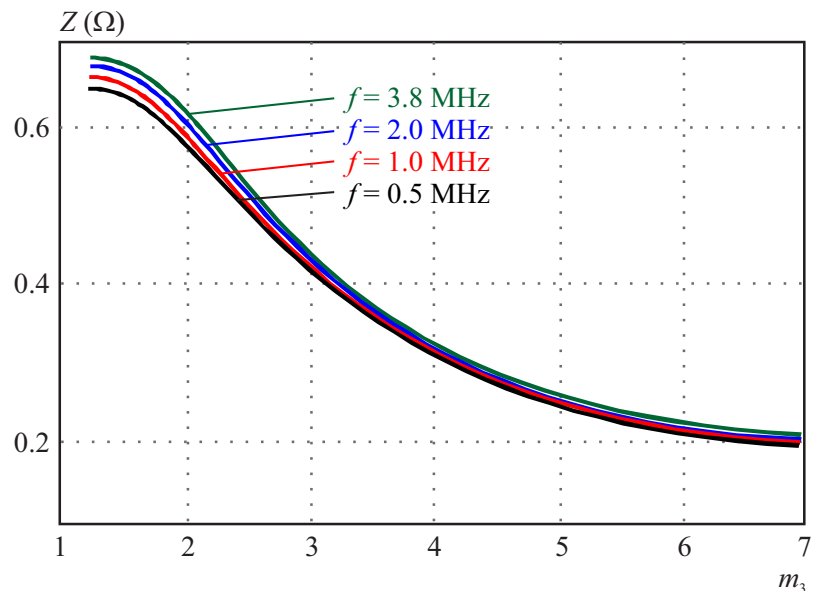

Fig. 10. Impedance as a function of resistivity ratio $m_{3}$, after the frequency normalization

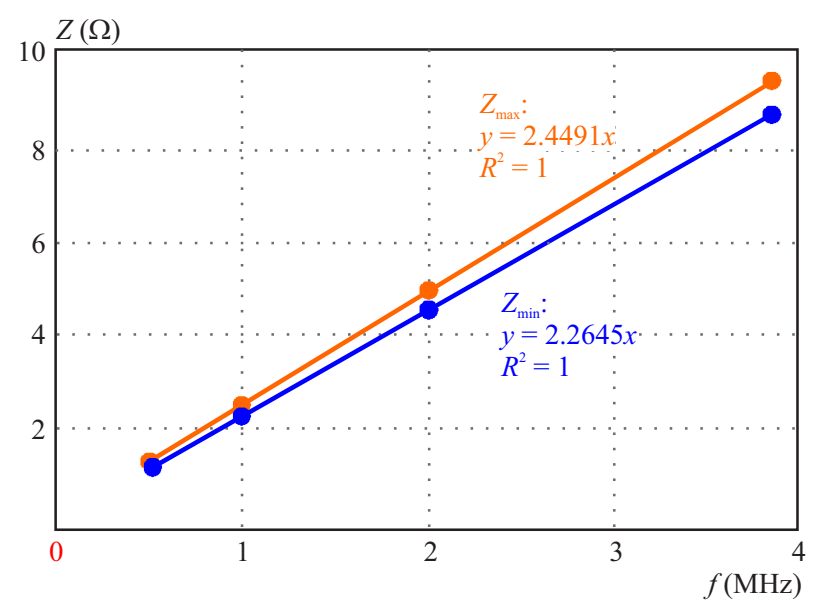

Fig. 12. Impedance-frequency data normalization

Table 2. Min/Max normalization values

\begin{tabular}{lcc}
\hline Parameter & $\min \left(x_{m}\right)$ & $\max \left(x_{m}\right)$ \\
\hline$P(\mathrm{~W})$ & $0.0112 f+0,01$ & $0.095 f+0.015$ \\
$Z(\Omega)$ & $2.2645 f$ & $2.4491 f$ \\
$\cos \varphi$ & 0.0058 & 0.0478 \\
$f(\mathrm{MHz})$ & 0.5 & 3.858 \\
$h_{2}(\mathrm{~mm})$ & 20 & 80 \\
$h_{1}(\mathrm{~mm})$ & 10 & 65 \\
\hline
\end{tabular}

The impedance and the power have to be frequency normalized before linear transformation, because of their strong dependency on frequency. Figures 9 and 10 show comparison of the impedance balance curves before and after frequency normalization, respectively.

The frequency normalization can be used only because the input data show almost a linear character on it, as Fig. 11 and 12 show. However, the dependencies are not possible to use for a unit recalculation, as in the case of the inductor current, because they are not truly linear and have different division ratio, dependent on parameter $m_{3}$. This is done by physical principle of induction melting, because every change of the melted material resistivity results in change of power and impedance distribution. 


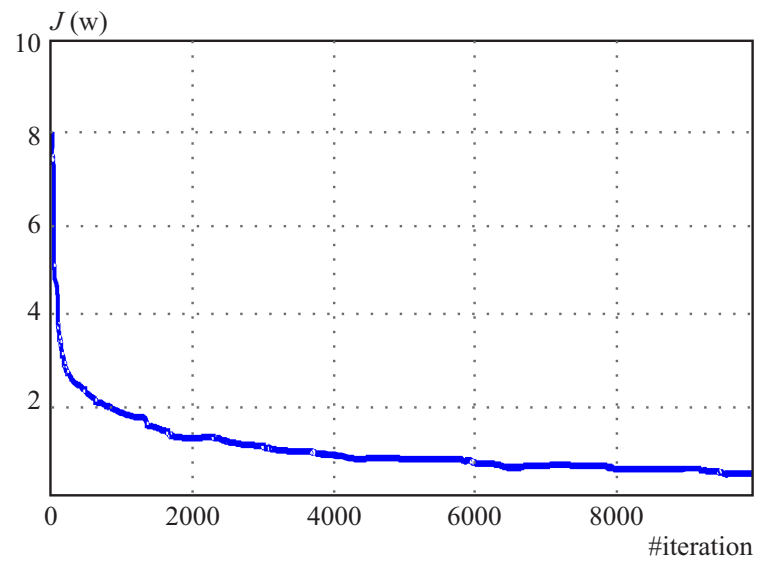

Fig. 13. Cost Function $J(w)$ during the iteration progress

At the end, the final calculation of the NN output is provided by activation values of the NN output layer. Activation value of each neuron can be calculated according (12), which shows how to calculate activation values for hidden layer. However, the values for the output layer are calculated in the same way, only the input parameters xi are replaced by activation values from previous layer $h_{i}$ and function $g$ represents the binary sigmoid function

$$
h_{i}=g\left(\sum_{i=1}^{N} w_{i} x_{i}\right) \text {. }
$$

\section{Experiment}

The NN learning process is rather time consuming, with strong dependency on number of elements in training set. For this reason the learning procedure has been divided into several stages and the result of every stage has been separately verified.

A first step used for verification of theoretical hypothesis and possibility of NN implementation for this kind of application contained only small portion of measured and interpolated data, based only on one geometrical layout and one operation frequency. The chosen geometrical parameter $h_{2}=50 \mathrm{~mm}, h_{1}=25 \mathrm{~mm}$ and frequency $f=2$ $\mathrm{MHz}$, while the other input parameters have been interpolated into 58 different values, based on parameter $m_{3}$. This training has been introduced to the algorithm and as the result of it, in a time $t=2.31 \mathrm{~min}$ and 10000 iterations NN with cost Function $J(w)=0.701$ was fully learned. The cost function waveform is shown in Fig. 13.

After this undemanding verification, it has been attempted to widen the training set for all calculated geometrical layouts on the same frequency $2 \mathrm{MHz}$.

The learning process took $t=31 \mathrm{~m}$ for next 15000 iterations, with final value of cost Function $J(w)=0.914$ and a result of it was fully learned $\mathrm{NN}$, which can reproduce every feature vector. Pre-calculated values from previous step have been used as initial values of weight coefficients $w$.

Next step contained interpolation of all results of MM calculations on the parameter $m_{3}$ for every calculated frequency. New training set contained 22330 feature vectors and training process took approximately 11 hours. Anyway, after that the NN has been able to correctly reproduce $99.588 \%$ of training set, while the 92 poorly reproduced values were only one quantization level away from the desired one. This mistake could be caused by poor interpolation, however after accepting one level quantization error (OLQE), the NN was able to fully reproduce the training set.

At this moment we left the training process off and began the phase of detailed testing. Every layout of MM result has been further interpolated in frequency, with $100 \mathrm{kHz}$ step, which gave us 215006 feature vectors containing 192676 sets which have never been introduced to NN during the learning process. This kind of test is very important for $\mathrm{NN}$, because only this way it can give us feedback concerning NN chosen dimensions. Results of the test show us $91.89 \%$ correctly reproduced output values, whereby after the OLQE acceptance this value reaches $99.99 \%$. Which means that only 4 values were interpreted with bigger error.

Analogous verification has been executed even for interpolated values of melted material height $h_{2}$ and inductor position $h_{1}$. In the case of $h_{2}$ test the inductor has been fixed in its lowest position, while melted material height has been interpolated in a range of $20-80 \mathrm{~mm}$ with $1 \mathrm{~mm}$ step, for every calculated frequency. This produced 10672 different set ups, which were reproduced correctly by NN with $84.633 \%$ probability, however after the OLQE acceptance this value reached $100 \%$. In the second case of $h_{1}$ test, melted material height was fixed at $80 \mathrm{~mm}$ and inductor position has been interpolated with $1 \mathrm{~mm}$ step. The percentage of absolutely correct results was $96.518 \%$ and with the OLQE acceptance 100\%.

Figure 14 shows activation of output neurons for randomly chosen input situation. In above described experimental verification a threshold function has been considered that transformed a neuron with the strongest output activation into a logical "1" and the others into a logical "0". However, a question for further investigation remains, whether it is possible to use for instance a parabolic approximation for obtaining a more accurate result.

\section{Discussion}

Performed implementation of real-time melted material resistivity measurement in the form of the NN shows very satisfying results. Not only was the NN able to learn the provided data from MM calculations with $100 \%$ accuracy, but also very satisfactory the interpolated data, which has never been introduced to it during the learning process. This is very important in comparison with the problem solution in a form of a look up table, which 


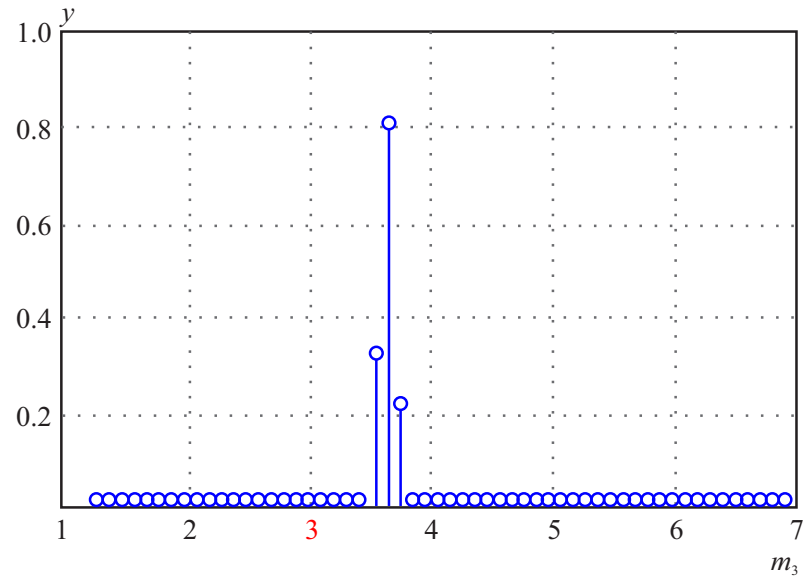

Fig. 14. Example of output neurons activation

would be able to provide accurate results only for stored feature vectors.

In addition to that, the NN implementation saves significant space in memory and therefore the final calculation can be performed by a microcontroller or, in our case, by FPGA without any memory utilization. The last advantage of this method is an inclusion of both power balance and impedance balance curves into a single solution, which means that there is no need to choose the more accurate one and its parallel implementation in the measurement system.

At the base of incorrectly reproduced results of $\mathrm{NN}$ has been found an uncertainty of measurement done by NN implementation. The uncertainty has very similar characteristics for each geometrical layout in dependency on frequency. Its waveform is shown at the figure 15 , for one layout and frequency.

The self determination of the uncertainty is strongly dependent on the chosen quantization step of parameter $m_{3}$, its actual value and operational frequency. The final function can be described, at $P=95 \%$ as

$$
u_{b}= \pm \frac{100 f}{\sqrt{3}}\left(\frac{4 r_{\text {melt }}^{2}}{50300^{2}\left(m_{3}\right)_{i}^{4}\left(m_{3}\right)_{i-2}^{4}}\right) \Omega \mathrm{cm}
$$

\section{Conclusion}

Performed method could be used in real time measurement system even with limited computational performance. However significant effort has to be made in preparation of different setups of MM and training of NN, which results has to be verified. Described method is also not applicable to the measurement of electrical resistivity of metals and alloys as the MM assumes a constant shape of the melt.

\section{Acknowledgements}

This paper was supported by the student research project SGS 2015031.

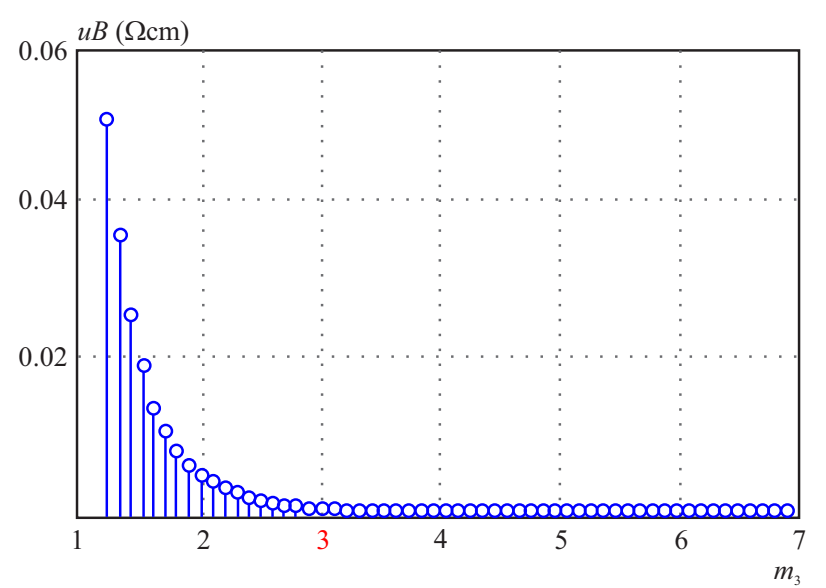

Fig. 15. Uncertainty of measurement, as a function of resistivity ratio $m_{3}$

\section{REFERENCES}

[1] B. Nacke, E. Baake, S. Lupi, F. Dughiero, M. Forzan, J. Barglik, A. Smalcerz, D. Dolega, A. Jakovics, S. Pavlovs, A. Aliferov and L. Goreva, "Theoretical background and aspects of electrotechnologies - Physical principles and realization", St. Petersburg, 2012, Publishing house of ETU.

[2] I. Poznyak and A. Petchenko, "Special Tool for Investigation and Controlling of Skull Melting Processes", International Scientific Colloquium on Modelling for Saving Resources, Riga, May 17-18, 2001.

[3] I. Poznyak, A. Petchenko and A. Shatunov, "Electrical conductivity measurement of oxides melts", International Scientific Colloquium on Modelling for Material Processing, Riga, June 8-9, 2006.

[4] A. N. Shatunov, A. I. Maksimov, A. Yu. Petchenko and I. Poznyak, "Methods of Electrical Resistivity Measurement for High-Temperature Melts", Inorganic Materials, 2011, vol. 47.

[5] I. Pozniak, A. Pechenkov, B. Nacke, T. And and Behrens, "Mathematical Modelling and Investigation of Induction System with Slated Crucible", Proceedings of the Int. Scient. Colloq. "Modelling for Electromagnetic Processing", Hannover, Germany, March 24-26, 2003, pp. 151 - 156.

[6] D. Shiffman, "The, Nature of Code USA, 2012 and ISBN-13, 978-0985930806, http://natureofcode.com/book/.

Received 6 October 2016

Pavel Votava (Ing) was born in the Czech Republic in 1987. He received a Master degree from the Faculty of Electrical Engineering at a University of West Bohemia in 2012. At the time he is a PhD student at the Department of Electric power engineering and Ecology and he is employed at Research Centre Rez, s.r.o.

Igor Poznyak (doc, Ing, CSc,) was born in the Russian Federation in 1964. He completed his PhD at the Saint Petersburg Electrotechnical University "LETI" in 1999 where he currently act as an associate professor. At the same time he is employed at Research Centre Rez, s.r.o. and University of West Bohemia. 